\title{
Global Enhancement but Local Suppression in Feature-based Attention
}

\author{
Norman Forschack ${ }^{1}$, Søren K. Andersen ${ }^{2}$, and Matthias M. Müller ${ }^{3}$
}

\begin{abstract}
A key property of feature-based attention is global facilitation of the attended feature throughout the visual field. Previously, we presented superimposed red and blue randomly moving dot kinematograms (RDKs) flickering at a different frequency each to elicit frequency-specific steady-state visual evoked potentials (SSVEPs) that allowed us to analyze neural dynamics in early visual cortex when participants shifted attention to one of the two colors. Results showed amplification of the attended and suppression of the unattended color as measured by SSVEP amplitudes. Here, we tested whether the suppression of the unattended color also operates globally. To this end, we presented superimposed flickering red and blue RDKs in the center of a screen and a red and blue RDK in the left and right periphery, respectively, also flickering at different frequen-
\end{abstract}

\section{INTRODUCTION}

While searching for a certain object in a cluttered environment, it seems beneficial that the object defining features are facilitated throughout the entire scene. Such global facilitation was proposed in the "guided search model" (Wolfe, 1994). Some years later, monkey intracranial recordings provided empirical evidence for such global facilitation of attended features, resulting in the "feature similarity gain model" (Martinez-Trujillo, 2011; Treue \& Martinez-Trujillo, 1999). In the meantime, a number of studies have confirmed the global effect of feature-based attention in the human brain (see also Andersen, Müller, \& Hillyard, 2011; Zhang \& Luck, 2009; Hayden \& Gallant, 2005; Hopf, Boelmans, Schoenfeld, Luck, \& Heinze, 2004). However, up to present only few studies have addressed the question whether featureselective attention also operates via suppression of distractor colors. Three recent color attention studies reported different results (Moher, Lakshmanan, Egeth, \& Ewen, 2014; Painter, Dux, Travis, \& Mattingley, 2014; Störmer \& Alvarez, 2014). Whereas Moher et al. (2014) found an inhibition of distractor colors with no global facilitation of the target color in a probe design, Painter et al. (2014), using

\footnotetext{
${ }^{1}$ Max-Planck-Institute for Human Cognitive and Brain Sciences, Leipzig, ${ }^{2}$ University of Aberdeen, ${ }^{3}$ University of Leipzig

cies. Participants shifted attention to one color of the superimposed RDKs in the center to discriminate coherent motion events in the attended from the unattended color RDK, whereas the peripheral RDKs were task irrelevant. SSVEP amplitudes elicited by the centrally presented RDKs confirmed the previous findings of amplification and suppression. For peripherally located RDKs, we found the expected SSVEP amplitude increase, relative to precue baseline when color matched the one of the centrally attended RDK. We found no reduction in SSVEP amplitude relative to precue baseline, when the peripheral color matched the unattended one of the central RDK, indicating that, while facilitation in feature-based attention operates globally, suppression seems to be linked to the location of focused attention. steady-state visual evoked potentials (SSVEPs), reported that target colors were facilitated (global effect) but distractor colors were not suppressed and this was restricted to conjunction search. Störmer and Alvarez (2014) also used frequency tagging to investigate suppressive interactions in color-based attention. Similar to Painter et al. (2014), they found SSVEP amplitudes to be greatest in the unattended visual field when color matched the target color (global facilitation). However, when color either matched the distractor color or was $30^{\circ}$ apart from the target color, SSVEP amplitudes were smallest. The authors interpreted their results as evidence for surround suppression in color space on the one and a global suppression of the to-be-ignored color on the other hand. The study by Störmer and Alvarez (2014) is based on a similar design as a previous own study (Andersen, Hillyard, \& Müller, 2013). In this study, we found intermediate SSVEP amplitudes when participants attended to opposite colors on the left and right side (i.e., the target color on one side was the distractor color on the opposite side and vice versa). This finding would seem consistent with global enhancement of attended and global suppression of unattended colors of roughly equal magnitude. However, we refrained from making such an interpretation, as the study lacked a valid baseline against which to assess enhancement and suppression. Whereas Painter et al. (2014) compared target/distractor colors against a neutral color, allowing valid conclusions about facilitation/suppression, Störmer and 
Alvarez (2014) compared colors of different similarity relative to the attended color and interpreted the reduction of SSVEP amplitudes as evidence for suppression. However, smaller SSVEP amplitudes per se do not allow drawing such a conclusion. To illustrate this: In our studies of spatial attentional shifts (Müller, 2008; Müller, Teder-Sälejärvi, \& Hillyard, 1998), stimuli in the left and right visual hemifield started to flicker before a cue instructed participants to shift attention to the left or right hemifield. We found greater SSVEP amplitudes for the attended compared with the unattended hemifield. To conclude that stimuli in the unattended hemifield were suppressed based on that amplitude difference would have been incorrect, because SSVEP amplitudes for the unattended stimulus did not change compared with the precue baseline and thus provided no indication of a suppressive effect after the onset of the cue. In one of our previous studies (Andersen \& Müller, 2010), we found suppression of the unattended color at centrally located and superimposed red and blue randomly moving dot kinematograms (RDKs) that flickered at two frequencies to elicit SSVEPs. In this study, we used a shifting paradigm, that is, participants were cued to shift attention to either red or blue, and we analyzed the SSVEP amplitude relative to a precue baseline. This study found enhancement of attended and suppression of unattended colors at the attended location; however, the pattern of results was consistent with suppression resulting from biased competition/divisive normalization (Reynolds \& Heeger, 2009; Desimone \& Duncan, 1995; Bundesen, 1990) of spatially overlapping stimuli. Thus, it did not allow drawing further conclusions of whether or not suppression acts throughout the visual field.

Neither the feature similarity gain model (Treue \& Martinez-Trujillo, 1999) nor the theory of visual attention (Bundesen, 1990) makes explicit assumptions as to whether feature-based attention operates through enhancement or suppression (but see Martinez-Trujillo \& Treue, 2004). However, both agree that feature-based attentional modulation acts throughout the visual field, a notion that is strongly supported by the finding that spatially global attentional selection of features is obligatory, that is, it occurs even when it explicitly conflicts with task demands (Andersen et al., 2013). Thus, both models agree that if feature-based attentional suppression occurs, it should be spatially global. Suppression can also occur through biased competition/divisive normalization between nearby stimuli, and there is general agreement that such suppression is spatially local (Reynolds \& Heeger, 2009; Desimone \& Duncan, 1995). A more recent model, the normalization model of attention (Reynolds \& Heeger, 2009), offers a formal implementation of biased competition (Desimone \& Duncan, 1995). In this model, attentional modulation is implemented through an "attention field," that is, a matrix of attentional gain values for all spatial locations and feature values by which sensory input is multiplied. In their model, Reynolds and Heeger (2009) implement the attention field through enhancement rather than suppression and with spatial and feature-based attention being orthogonal (thus featurebased attention is spatially global). However, these constraints are not explicit in the model, and thus, the model would be consistent with any combination of spatially global or local enhancement and suppression. The present experiment used our shifting paradigm (Andersen \& Müller, 2010) to obtain a precue baseline of SSVEP amplitudes while also measuring feature-based attentional modulation at spatially unattended locations. Importantly, stimuli at unattended locations were nonoverlapping, so that any observed effects can unambiguously be attributed to global mechanisms of feature selection rather than also being influenced by biased competition with superimposed stimuli. We found global facilitation of the attended color, but suppression of the unattended color was restricted to the attended central location. Thus, whereas facilitation acts globally, suppression seems to be restricted to the attended location.

\section{METHODS}

\section{Participants}

After receiving information about the experiment's nature, 23 healthy participants (10 women, 4 left-handed) with normal color vision and normal or corrected-tonormal visual acuity at the age of 20-33 years (average $=$ 24.2 years) gave their written consent. All participants were included in the analyses. The experiment was performed in accordance to the Code of Ethics of the World Medical Association and the local ethics committee.

\section{Stimuli and Procedure}

We presented two overlapping red and blue RDKs centered on a fixation cross in the center of a 19-in. cathode ray tube display and two additional RDKs, one on each side in the periphery of the screen being red or blue (see Figure 1). Screen resolution was set to 640-by- 480 pixel, color depth to 32 bits per pixel, and refresh rate to $120 \mathrm{~Hz}$. Participants viewed stimuli at a distance of $80 \mathrm{~cm}$, resulting in a vertical and horizontal size of $9.72^{\circ}$ by $4.87^{\circ}$ of visual angle, respectively. Central and peripheral RDKs were separated by the width of a single RDK (i.e., $4.87^{\circ}$ visual angle; see Figure 1). Each RDK consisted of 75 randomly and independently moving quadratic dots with an edge size of $0.325^{\circ}$ of visual angle, translating with a speed of $0.051^{\circ}$ per frame. Dots were drawn in random order to prevent depth cues. Each RDK flickered with a specific frequency (Figure 1, from left to right: $12.5,15,17.5$, and $10 \mathrm{~Hz}$ ) to allow for the analysis of distinct SSVEPs. Stimulation was synchronized to the monitor's refresh rate, and an interpolation technique was employed to elicited SSVEPs at 12.5 and $17.5 \mathrm{~Hz}$ (Andersen \& Müller, 2015). The RDKs were presented by the pure red and blue phosphors of the monitor to 


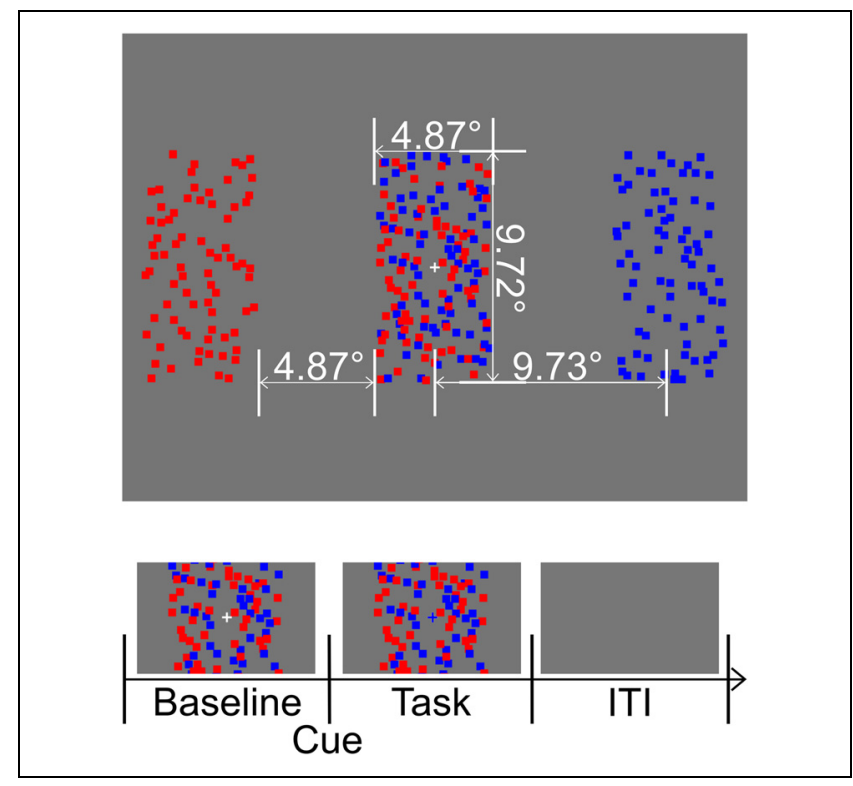

Figure 1. Depiction of stimulus display, stimulus dimensions in degree of visual angle and trial structure. Trials started with the simultaneous presentation of a white fixation cross together with a total of 150 red and 150 blue spatially intermingled dots, which were in continuous random motion. In addition, red dots flickered at $12.5 \mathrm{~Hz}$ (periphery) and $15 \mathrm{~Hz}$ (center), and blue dots flickered at $10 \mathrm{~Hz}$ (periphery) and $17.5 \mathrm{~Hz}$ (center). The color of the central fixation cross changed after a random interval of $1250-1750 \mathrm{msec}$ to red or blue, thereby indicating the task-relevant central dots. Within the subsequent time period of $1783 \mathrm{msec}$, participants discriminated brief coherent motions of a subset of dots of the attended from the unattended color. This was followed by a blank gray screen for $1800 \mathrm{msec}$, serving as a break between trials and then a new trial started. ITI $=$ intertrial interval.

produce highly saturated colors against the gray background. Their luminance was individually adjusted for isoluminance to the gray background $\left(5.8 \mathrm{~cd} / \mathrm{m}^{2}\right)$ by means of heterochromatic flicker photometry (Wagner \& Boynton, 1972). This fairly low luminance of the background was chosen to ensure that the subjectively isoluminant blue for all participants did not exceed the maximum luminance of the blue phosphor. Frequencyspecific RDKs remained at the same position for all participants. To control for possible visual field biases and, thus, preferred processing of a certain color, we swapped peripheral colors for a subset of six participants without changing stimulation frequencies.

In all trials, presentation of four flickering RDKs together with a white fixation cross started simultaneously. After a random interval of 1250-1750 msec, the fixation cross changed color to blue or red (cue) until the end of the trial, lasting $1783 \mathrm{msec}$. This was followed by an ISI of 1800 msec during which only the gray background was presented. The interval before the color change of the fixation cross served to establish a stable steady-state response and was used to calculate the respective baseline per experimental condition.

With cue onset and for $1500 \mathrm{msec}$, a subset of dots of the central RDKs (60\% to avoid tracking of individual dots) could engage in a transient $(300 \mathrm{msec})$ coherent motion in one of two cardinal directions (up- or downwards). Participants were instructed to always fixate the cross and to press the space bar as fast as possible, as soon as they recognized a coherent motion onset within the RDK of the attended color (target), but to ignore coherent motion onsets within the unattended RDK (distractor). For each trial, any combination of up to two targets or distractors was possible. The onset of subsequent coherent motion events was separated by at least $700 \mathrm{msec}$. Importantly, dots in the peripheral RDKs never showed coherent motion events, and participants were instructed to always ignore them.

The experiment consisted of 480 trials in total, 240 for each cue condition, presented in random order. In 240 trials, there was no coherent dot motion in any central $\mathrm{RDK}$. The remaining trials were further divided in 120 trials having one and 120 trials having two targets and/or distractors in one of the central RDKs, that is, in sum 90 targets and 90 distractors were presented in both "attend red" and "attend blue" conditions. Over all trials, events were uniformly distributed over 10 windows of $150-\mathrm{msec}$ length each, to allow for a time resolved analysis of the behavioral data.

During a short break after each block of 60 trials ( $\sim 5 \mathrm{~min})$, participants received feedback about their current performance. Participants started the next block self-paced via button press. Before recording the experimental blocks, participants performed a training session of four or more blocks of 30 trials each until they reached stable performance of at least $70 \%$ hit rates and less than 10\% false alarms.

\section{Behavioral Data}

Only button presses that occurred between 250 and 900 msec after the onset of a target or distractor event were considered as hits or false alarms, respectively. Similar to our previous study (Andersen \& Müller, 2010), RTs were summarized and averaged in 10 sequential time bins of 150 msec each. Successive bins were compared by means of two-tailed paired $t$ tests to determine the point at which maximal performance was reached. Within-subject confidence intervals were calculated by subtracting individual mean behavioral performance over all bins (Loftus \& Masson, 1994). The small amount of behavioral data samples per bin did not allow for reliable computation of the sensitivity parameter $d^{\prime}$ (Wickens, 2002).

\section{EEG Recording and Analysis}

Brain electrical activity was recorded at a sampling rate of $256 \mathrm{~Hz}$ from $64 \mathrm{Ag} / \mathrm{AgCl}$ electrodes mounted in an elastic cap using an ActiveTwo amplifier system (BioSemi, Amsterdam, The Netherlands). We monitored lateral and vertical eye movements by placing two additional 
bipolar montages at the outer canthi (horizontal EOG) and above and below the right eye (vertical EOG), respectively.

EEG data analysis was performed offline using the EEGLAB toolbox (Delorme \& Makeig, 2004) and custombuilt MATLAB scripts (The MathWorks, Natick, MA). We extracted epochs from -1000 to $2000 \mathrm{msec}$ relative to the shifting cue onset only including trials without targets or distractors to ensure that selective attention was based on color only, with no interference from coherent motion events. The first and last $200 \mathrm{msec}$ of each epoch were required to attenuate edge artifacts of the Gabor filter applied for time-frequency analysis. Thus, before averaging, the time window between -800 and $1800 \mathrm{msec}$ relative to the cue passed a semiautomatic preprocessing pipeline to clean trials from artifactual data, resulting in an average rejection rate of $14.2 \%$ trials. As a first step, the mean across the entire epoch and any linear trends were subtracted from each epoch. After removing trials with blinks and eye movements of more than $2^{\circ}$ of visual angle (i.e., $18 \mu \mathrm{V}$ ), "statistical control of artifacts in dense array EEG/MEG studies" (Junghöfer, Elbert, Tucker, \& Rockstroh, 2000) was applied. This procedure combines trial exclusion and channel approximation based on statistical parameters of the data. Trials with more than 10 contaminated neighboring electrodes in a particular scalp region were excluded from further analysis. Subsequently, data was re-referenced to average reference, and trials were averaged for each experimental condition.

Iso-contour voltage maps of the $10-\mathrm{Hz}$ (blue, periphery), $12.5-\mathrm{Hz}$ (red, periphery), $15-\mathrm{Hz}$ (red, center), and $17.5-\mathrm{Hz}$ (blue, center) mean SSVEP amplitudes averaged across experimental conditions were calculated by means of discrete Fourier transform to determine the appropriate electrode cluster for statistical analysis (see Figure 3A). For central RDKs, SSVEP amplitudes were maximal at occipital electrodes around $\mathrm{Oz}$ and clearly above noise level (Figure 3B). For the 10- and 12.5-Hz flickering peripheral RDKs, this was true in the area of electrodes P9 and P10, respectively. Subsequent analyses of SSVEP amplitude were thus based on the averaged amplitude of four neighboring electrodes centered around $\mathrm{Oz}$, PO7, or PO8, respectively. Cluster spectra were calculated by means of a Fourier transform (Matlab fft function), with zero-padding to extend each electrode time series to $16,384\left(=2^{14}\right)$ points. The absolute value of the resulting complex Fourier coefficients was multiplied by two and divided by the number of sampling points in each epoch (before zero-padding) to scale the result in microvolt.

In a mixed effect ANOVA design, we tested whether swapping blue/red of the peripheral RDKs had any effect on SSVEP amplitude and found neither a significant main effect of between-subject factor Color side $(F(1,21)<1)$ nor a significant interaction with the factor of Attention and $\mathrm{RDK}$ position $(F(3,63)=1.36, p=.26)$. In addition, we tested possible differences of SSVEP amplitudes in the left or right peripheral RDK and also found no significant effects (main effect Hemifield and interaction with Attention $(F(1,22)<1)$. Because of this, we pooled SSVEP amplitudes across the two central and the two peripheral stimuli separately (see below).

To analyze the time course of the SSVEP amplitudes, we calculated frequency-specific amplitude envelopes using a Gabor filter (Gabor, 1946) having equal frequency resolution of $\pm 0.9 \mathrm{~Hz} \mathrm{FWHM}$ and temporal resolution of \pm 245 msec FWHM. Compared with our previous study (see also Andersen \& Müller, 2010), frequency resolution was increased to avoid cross-talk between the analyzed frequencies, which in turn decreased the temporal resolution, respectively. Given that we found no differences in SSVEP amplitudes for the two peripheral RDKs, we pooled data across the two central and the two peripheral frequencies separately after normalization. Normalization was performed by dividing the amplitudes for each data point by the averaged amplitude of a baseline from -400 to $-300 \mathrm{msec}$ before the cue and subsequently subtracting 1.0 . This baseline window was chosen to be similar to our previous study (Andersen \& Müller, 2010) but started $100 \mathrm{msec}$ earlier to prevent the baseline from being contaminated by the postcue interval due to the lower temporal resolution of the Gabor filter in the present experiment. To verify that our findings do not depend upon the particular choice of baseline, we recalculated the analysis with a baseline ranging from -550 to $-250 \mathrm{msec}$ (this is the baseline of maximal duration as it keeps one filter width separation from the beginning of the artifact free time range ( $-800 \mathrm{msec})$ and cue onset $(0 \mathrm{msec})$. We did not observe any qualitative differences with this longer baseline and thus proceeded all further analyses with the -400 to $-300 \mathrm{msec}$ baseline. For statistical analysis of SSVEP amplitudes, these baseline-corrected time courses were averaged over a time range of $250-800 \mathrm{msec}$ postcue and subjected to a $2 \times 2$ repeated-measures ANOVA with factors of Location (RDK position central vs. peripheral) and Attention (attended vs. unattended). The starting point was determined to not include signal from the precue baseline period due to the temporal smearing of the Gabor kernel, that is, $245 \mathrm{msec}$. The endpoint was chosen based on our previous study with a similar design but only having the central RDKs in which we found the maximum amplitude differences up to $800 \mathrm{msec}$ after shifting cue onset (Andersen \& Müller, 2010), which visually matched the current SSVEP amplitude time courses. ANOVA statistics and the bootstrapped confidence intervals (conditionwise resampling of subject indices with 10,000 iterations) were computed in R (R Core Team, 2012) with the ezPackage developed by Mike Lawrence (2013, version 4.2-2, https:/github.com/mike-lawrence/ez). Post hoc two-tailed $t$ tests with a significance level of $p<.05$ were applied where indicated. Effect sizes were quantified as generalized eta-squared ( $\eta_{G}^{2}$; Bakeman, 2005). 


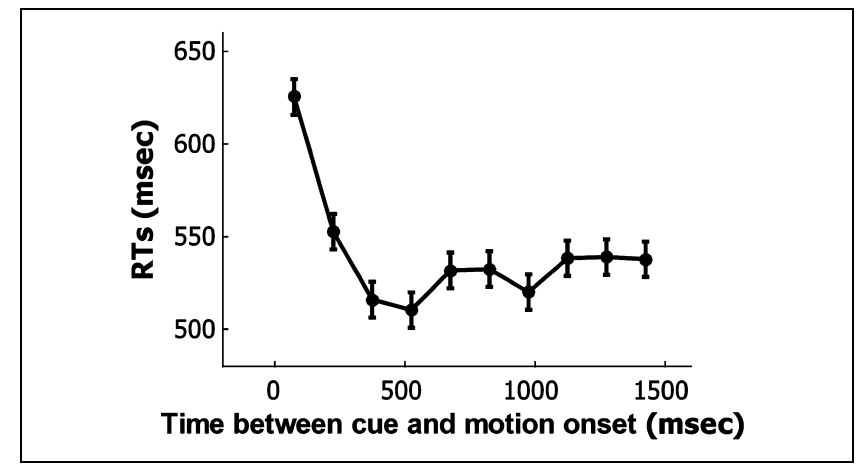

Figure 2. RTs of detected coherent motion events as a function of time after cue onset. RTs dropped until the third time bin (300-450 msec) and increased again during the fifth time bin (600-750 msec).

\section{RESULTS}

\section{Behavioral Performance}

The average hit rate was $84.0 \%(S D=5.9 \%)$ with average false alarm rates of $15.7 \%(S D=1.8 \%)$. Thus, the task was challenging, but participants performed well above chance. RTs $(M=540.7 \mathrm{msec}, S D=47.2 \mathrm{msec})$ decreased significantly from first $(0-150 \mathrm{msec})$ to second $(150-300 \mathrm{msec})$ and second to third (300-450 msec) time bin $(t(22)=$ $10.64, p<.001$ and $t(22)=4.74, p<.001$, respectively; see Figure 3). We found an increase in RTs from fourth $(450-600 \mathrm{msec})$ to fifth time bin $(600-750 \mathrm{msec} ; t(22)=$ $-4.65, p<.001)$. All following time bins did not differ significantly from time bin 5 (Figure 2).

\section{SSVEP Amplitudes}

As depicted in Figure 3A, SSVEP amplitudes of the central RDKs showed a narrow peak at central occipital sites (O1, $\mathrm{Oz}, \mathrm{Iz}, \mathrm{O} 2)$, whereas the peripheral RDKs showed maximum amplitudes at more lateralized occipital electrodes contralateral to their respective visual hemifield location (P7, P9, PO7, PO3 vs. P8, P10, PO8, PO4, respectively). As described above, three clusters of four electrodes each were chosen and averaged for subsequent analysis. As depicted in the respective spectrum in Figure 3B, SSVEP amplitudes were greater when the respective RDK was attended compared with when it was not. When a peripheral RDK matched color with the centrally attended RDK, higher grand-averaged SSVEP amplitudes were observed at respective lateral occipital clusters compared with when colors did not match.

Figure 4 depicts the time course of SSVEP amplitudes for central and peripheral RDKs. Identical to our previous study (Andersen \& Müller, 2010), attention to the color of the centrally displayed RDKs resulted in an increase of SSVEP amplitudes, whereas the unattended color was suppressed, relative to the precue baseline. This was different in the peripherally located RDKs that exhibited an SSVEP amplitude increase when color matched the centrally attended one, with no difference in amplitudes relative to precue baseline when the color matched the centrally unattended one.

This was confirmed by the statistical analysis of the baseline-corrected averaged SSVEP amplitude in the time window between 250 and $800 \mathrm{msec}$ after shifting cue
Figure 3. (A) Spline-interpolated isocontour maps of the grand-averaged SSVEP amplitudes of the averaged attentional conditions. Circles around electrode locations indicate the cluster of electrodes used for statistical analysis of the respective frequencies. (B) Grand-averaged amplitude spectrum obtained by Fourier analysis of SSVEP waveforms of the entire stimulation period elicited under the two attentional conditions averaged across the frequency specific electrodes. Peak amplitudes are located at the four stimulation frequencies.

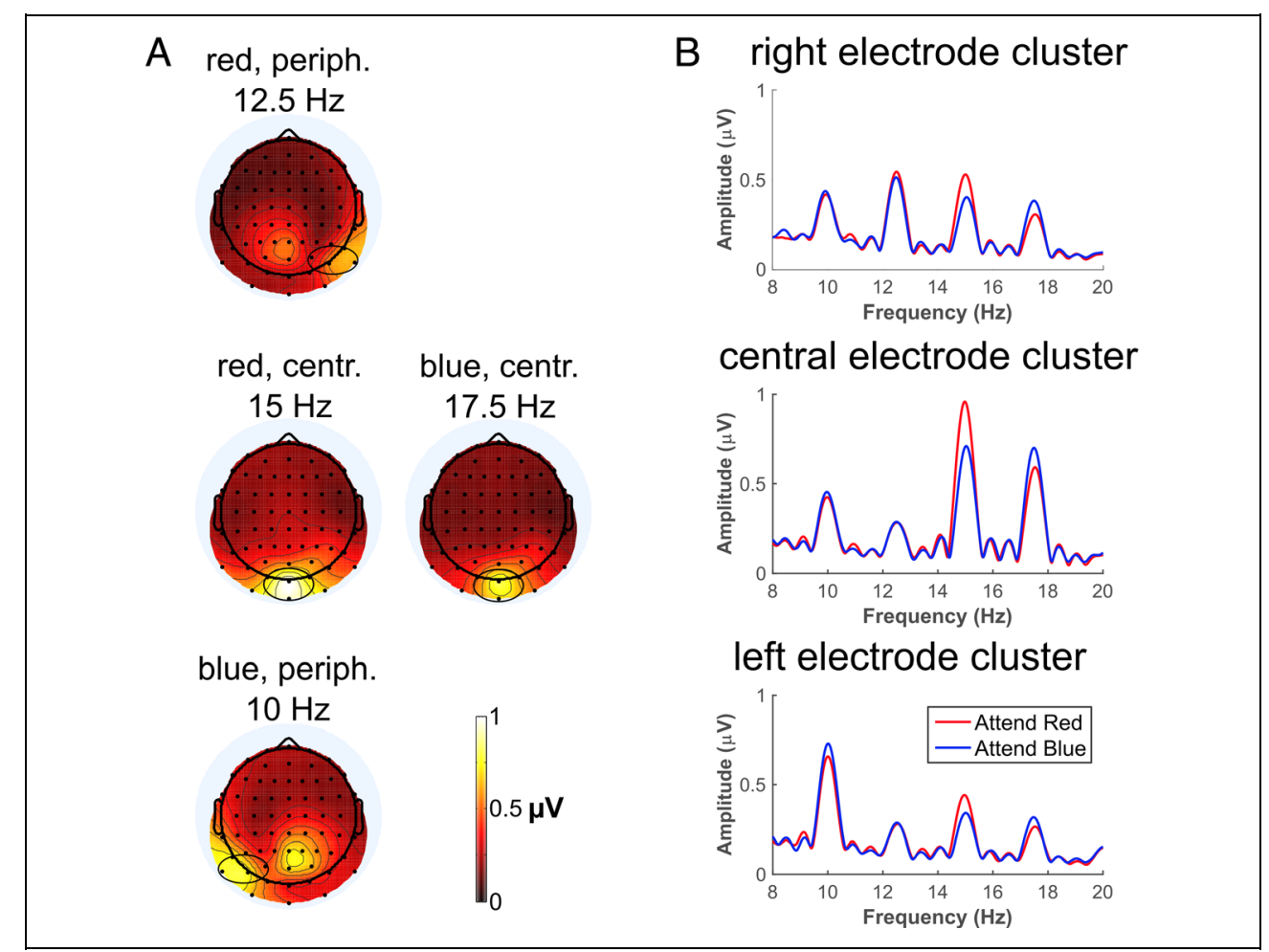


Figure 4. Grand-averaged time courses of SSVEP amplitudes (bold lines), averaged across clusters of electrodes showing the strongest amplitude in the frequencies of the respective RDKs (see Figure 3). After normalization to -400 to -300 msec precue baseline, time courses were pooled for red and blue dots. (left) Time courses of centrally presented RDKs differentiate after cue onset: SSVEP signal amplitudes become enhanced for the attended color and suppressed for the unattended color, which replicates the main finding of Andersen and Müller (2010). (right) Time courses of peripherally presented RDKs show enhanced SSVEP signal amplitude only if the color of

the peripheral RDK matches the color of the centrally attended RDK. Note that thin lines represent $95 \%$ confidence intervals for the attended (solid) and unattended condition (dashed). Grayed background resembles baseline used for normalization. Temporal resolution of the Gabor filter is $\pm 245 \mathrm{msec}$.

onset. We found a significant main effect of Attention ( $F(1$, $\left.22)=34.19, p<.00001, \eta_{G}^{2}=0.24\right)$ as well as a significant Attention $\times$ Location interaction $(F(1,22)=5.23, p=.03$, $\left.\eta_{G}^{2}=0.04\right)$. The main effect of Location was not significant $\left(F(1,22)=1.49, p=.24, \eta_{G}^{2}=0.026\right)$. As depicted in Figure 5, the significant interaction was driven by a lack of suppression for SSVEP amplitudes of peripheral RDKs when their color did not match the color of the centrally attended RDKs as confirmed by post hoc $t$ tests against zero (central RDK-color attended: $t(22)=6.26$, $p<.00001$; central RDK-color unattended: $t(22)=-6.35$,

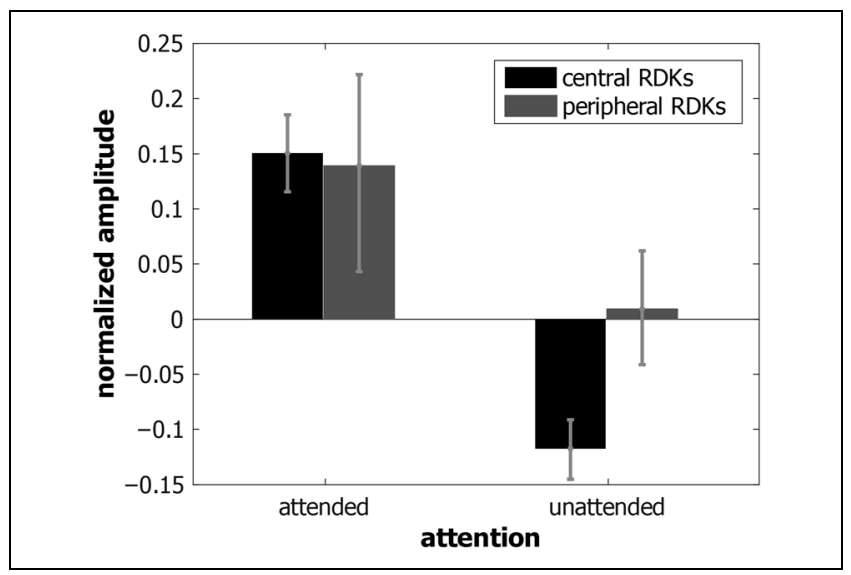

Figure 5. SSVEP amplitudes obtained by averaging Gabor time courses from 250 to 800 msec after cue onset. The differential SSVEP amplitude pattern of enhancement and suppression between RDK locations is evident in the interaction term and can readily be seen by examining the $95 \%$ confidence intervals obtained by bootstrapping conditions across subjects 10,000 times (see Methods). $p<.00001$; peripheral RDK-color attended: $t(22)=2.32$, $p=.03$; peripheral RDK-color unattended: $t(22)=0.27, p=$ .79). Importantly, unattended color stimuli elicited significantly lower amplitudes at the central than at peripheral locations $(t(22)=2.80, p=.01)$.

\section{DISCUSSION}

Does suppression of an unattended color act globally throughout the visual field? We found that, although facilitation of the attended color acted globally, suppression of the unattended color was restricted to the centrally located RDK where attention was focused to perform a target detection task. Our results confirmed both (a) global enhancement of neural processing of the attended color as predicted by the feature similarity gain model (Treue \& Martinez-Trujillo, 1999) and reported in many other studies and (b) suppression of the unattended color in centrally presented RDKs when participants were cued to shift attention to one color (Andersen \& Müller, 2010).

Given that we had four different frequencies in the present stimulus display, temporal resolution of the Gabor filters needed to be decreased $( \pm 245 \mathrm{msec})$ compared with our previous study ( $\pm 183 \mathrm{msec}$ ) to avoid spectral leakage between stimulation frequencies (Andersen \& Müller, 2010). Therefore, a more fine detailed analysis of the temporal dynamics was not in the focus of this study. Nevertheless, amplitude enhancement and suppression reached their maximum around $600 \mathrm{msec}$ postcue onset. Similar to our previous study (Andersen \& Müller, 2010), behavioral data followed this time course, that is, maximal performance was reached in that time range. In that 
previous study, we also tested that participants were in a "neutral" attentional state during the baseline period and excluded the possibility that participants maintained attention to the color that was cued in the preceding trial. In this study, we used the identical design with unpredictable shifting cues; thus, we are confident that participants were in a "neutral" attentional state during baseline.

Our finding of facilitation and suppression at the attended location can be explained by competitive interactions between the two RDKs as predicted by the "biased competition" account (Desimone \& Duncan, 1995). The closely intermingled attended and unattended color dots are likely to fall within the same receptive fields of color processing neurons, which is exactly the condition when competition is greatest, leading to a suppression of the unattended color (Chelazzi, Miller, Duncan, \& Desimone, 2001; Luck, Chelazzi, Hillyard, \& Desimone, 1997). In a behavioral study, White and Carrasco (2011) presented a dual task in which participants were cued to attend to one location with superimposed stimuli (motion or orientation). In the to-be-ignored location, two competing stimuli were presented and participants were asked to indicate whether or not they observed a coherent feature as the cued one in the to-be-attended location (secondary task). Similar to our findings, results clearly indicated that task performance in the secondary task benefited more from the facilitation of cued features than from suppression of mismatching features.

Contrary to the studies by Störmer and Alvarez (2014) and Painter et al. (2014), in this study, postcue SSVEP amplitude enhancement or suppression was assessed relative to a precue baseline. The fact that attended and unattended colors differ in SSVEP amplitudes does not allow one to conclude that the to-be-ignored color was actively suppressed, as illustrated in the Introduction section. In the Painter et al. (2014) study, participants performed a conjunction search for targets in the cued color among distractors, while task-irrelevant checkerboards in the periphery could either match the target, distractor, or a neutral color. Although the logic of referencing effects against a neutral color is different from our present study, both studies found an enhancement of attended, but no suppression of unattended, colors at spatially unattended locations. Both types of comparison levels may be affected by different biases: Participants are effectively performing a cue detection task during the baseline interval, which has different attentional demands from the postcue interval. On the other hand, task-irrelevant neutral stimuli might still interfere with task performance (e.g., as additional noise), and this could affect how attention is allocated to them. ${ }^{1}$ Also, it is not always possible to match neutral stimuli physically with attended and unattended stimuli, which might bias the results unless additional controls are performed (e.g., Moher et al., 2014). Thus, the convergence of the present findings with those of Painter et al. (2014), which rely on baseline or neutral stimulus comparisons, respec- tively, provides good evidence for a global feature-based enhancement.

Interestingly, Painter et al. (2014) reported of no global SSVEP amplitude enhancement during unique-feature search and interpreted this as evidence that attentional enhancement was only global in the conjunction search conditions of their experiment. This seems at odds with our present and previous (Andersen et al., 2013; Andersen, Fuchs, \& Müller, 2011) observations of global feature-based attention effects when attentional selection is based on a single feature (color). Furthermore, in a recent study attentional selection of feature conjunctions was simply the sum of attending the constituent features separately (Andersen, Müller, \& Hillyard, 2015), that is, attentional selection of color is independent of attentional selection on another feature dimension. These findings question Painter et al.'s (2014) claim of spatially local feature-based enhancement in single feature search. As pointed out by Foster and Adam (2014), it is very likely that participants did not need a feature search strategy, because the display was a typical odd-one-out display that does not require search but operates on a preattentive stage (Wolfe, 1994; Treisman \& Gelade, 1980) that does not require global facilitation. Unlike the present paradigm, Painter et al. (2014) did not measure feature-based attentional enhancement at the attended location and thus cannot exclude the possibility that there was no feature-based attentional enhancement at all in their single feature search conditions.

In two probe studies with a similar design (Moher et al., 2014; Zhang \& Luck, 2009), the authors came to different conclusions with regard to neural mechanisms of unattended stimuli in feature-based attention. Both studies presented probes in either the same or different color as the attended one at the ignored visual hemifield and measured the P1 amplitude to the probes. Zhang and Luck (2009) interpreted amplitude differences in the P1 between attended and unattended color as evidence for global facilitation, but their study contained neither a precue baseline nor a neutral stimulus comparison level. Moher et al. (2014) introduced a third probe color that was not present in the attended location. Relatively to this neutral color, P1 probe amplitudes of the ignored color were decreased with no difference to the target color. They concluded that feature-based attention acts on global suppression of the ignored color. However, Painter et al. (2014) also used a neutral color in their conjunction search trials but found no differences in SSVEP amplitudes between the neutral and ignored colors. In the present experiment and those of Painter et al. (2014), the assignment of attended and unattended colors switched between trials. In addition, the neutral color was switched between blocks in Painter et al. (2014). By comparison, Moher et al. (2014) used a fixed assignment of colors (attended/unattended/neutral) for each participant and counterbalanced this assignment between participants. Thus, it could be that the suppression 
observed by Moher et al. (2014) builds up on a much slower timescale and can therefore only be observed when attentional settings are kept constant across the experiment. This is much different from the spatially local suppression of the unattended color at the attended location that occurred with trial-by-trial cueing in the present experiment and which we interpret as a consequence of biased competition (Desimone \& Duncan, 1995).

Would a suppression of unattended peripheral RDKs have been observed if they had been presented together with superimposed stimuli of the attended color? We here avoided such a design to be able to clearly interpret any modulation of processing of peripheral RDKs as being due to global feature-based attention rather than biased competition (Desimone, 1998; Desimone \& Duncan, 1995) with spatially superimposed stimuli. A previous study using superimposed stimuli both inside and outside the spatial focus of attention observed feature-based attentional modulation of comparable magnitude at attended and unattended locations (Andersen, Fuchs, \& Müller, 2011). Thus, although this study lacked a precue baseline, its results indicate that a suppression of unattended color stimuli at unattended locations may result as a consequence of biased competition with superimposed attended color stimuli, as otherwise feature-based attentional modulation on the unattended side would have been smaller than on the attended side. However, additional research would be necessary to directly test this proposition.

In summary, in our attention shifting experiment that uses a precueing baseline to investigate the neural underpinnings of global feature based attention, we found spatially global facilitation of the attended color but only local suppression of the unattended color constrained to the attended location. It is likely that this suppression of unattended color stimuli observed here is due to local biased competition with superimposed attended color stimuli rather than global feature-based attention.

Reprint requests should be sent to Matthias M. Müller, Institute of Psychology, University of Leipzig, Neumarkt 9-19, 04109 Leipzig, Germany, or via e-mail: m.mueller@uni-leipzig.de.

\section{Note}

1. Ideally, the "neutrality" of such stimuli in terms of task performance could be assessed by comparing performance with control conditions in which the neutral stimuli are physically removed

\section{REFERENCES}

Andersen, S. K., Fuchs, S., \& Müller, M. M. (2011). Effects of feature-selective and spatial attention at different stages of visual processing. Journal of Cognitive Neuroscience, 23, $238-246$.

Andersen, S. K., Hillyard, S. A., \& Müller, M. M. (2013). Global facilitation of attended features is obligatory and restricts divided attention. Journal of Neuroscience, 33, 18200-18207.
Andersen, S. K., \& Müller, M. M. (2010). Behavioral performance follows time-course of neural facilitation and suppression during cued shifts of feature-selective attention. Proceedings of the National Academy of Sciences, U.S.A., 107, 13878-13882.

Andersen, S. K., \& Müller, M. M. (2015). Driving steady-state visual evoked potentials at arbitrary frequencies using temporal interpolation of stimulus presentation. $B M C$ Neuroscience, 16, 95.

Andersen, S. K., Müller, M. M., \& Hillyard, S. A. (2011). Tracking the allocation of attention in visual scenes with steady state evoked potentials. In M. I. Posner (Ed.), Cognitive neuroscience of attention (2nd ed., pp. 197-216). New York: The Guilford Press.

Andersen, S. K., Müller, M. M., \& Hillyard, S. A. (2015). Attentional selection of feature conjunctions is accomplished by parallel and independent selection of single features. Journal of Neuroscience, 35, 9912-9919.

Bakeman, R. (2005). Recommended effect size statistics for repeated measures designs. Behavior Research Methods, 37, 379-384.

Bundesen, C. (1990). A theory of visual attention. Psychological Review, 97, 523-547.

Chelazzi, L., Miller, E. K., Duncan, J., \& Desimone, R. (2001). Responses of neurons in macaque area V4 during memory-guided visual search. Cerebral Cortex, 11, 761-772

Delorme, A., \& Makeig, S. (2004). EEGLAB: An open source toolbox for analysis of single-trial EEG dynamics including independent component analysis. Journal of Neuroscience Methods, 134, 9-21.

Desimone, R. (1998). Visual attention mediated by biased competition in extrastriate visual cortex. Philosophical Transactions of the Royal Society of London, Series B, Biological Sciences, 353, 1245-1255.

Desimone, R., \& Duncan, J. (1995). Neural mechanisms of selective visual attention. Annual Review of Neuroscience, 18, 193-222.

Foster, J. J., \& Adam, K. C. (2014). Is feature-based attention always spatially global during visual search? Journal of Neuroscience, 34, 8662-8664.

Gabor, D. (1946). Theory of communication. Proceedings of the Institute of Electrical Engineers, 93, 429-441.

Hayden, B. Y., \& Gallant, J. L. (2005). Time course of attention reveals different mechanisms for spatial and feature-based attention in area V4. Neuron, 47, 637-643.

Hopf, J.-M., Boelmans, K., Schoenfeld, M. A., Luck, S. J., \& Heinze, H.-J. (2004). Attention to features precedes attention to locations in visual search: Evidence from electromagnetic brain responses in humans. Journal of Neuroscience, 24, 1822-1832.

Junghöfer, M., Elbert, T., Tucker, D. M., \& Rockstroh, B. (2000). Statistical correction of artifacts in dense array EEG/MEG studies. Psychophysiology, 37, 523-532.

Loftus, G. R., \& Masson, M. E. (1994). Using confidence intervals in within-subject designs. Psychonomic Bulletin \& Review, 1, 476-490.

Luck, S. J., Chelazzi, L., Hillyard, S. A., \& Desimone, R. (1997). Neural mechanisms of spatial selective attention in areas $\mathrm{V} 1, \mathrm{~V} 2$, and V4 of macaque visual cortex. Journal of Neurophysiology, 77, 24-42.

Martinez-Trujillo, J. (2011). Searching for the neural mechanisms of feature-based attention in the primate brain. Neuron, 70, $1025-1028$.

Martinez-Trujillo, J. C., \& Treue, S. (2004). Feature-based attention increases the selectivity of population responses in primate visual cortex. Current Biology, 14, 744-751. 
Moher, J., Lakshmanan, B. M., Egeth, H. E., \& Ewen, J. B. (2014). Inhibition drives early feature-based attention. Psychological Science, 25, 315-324.

Müller, M. M. (2008). Location and features of instructive spatial cues do not influence the time course of covert shifts of visual spatial attention. Biological Psychology, 77, 292-303.

Müller, M. M., Teder-Sälejärvi, W., \& Hillyard, S. A. (1998). The time course of cortical facilitation during cued shifts of spatial attention. Nature Neuroscience, 1, 631-634.

Painter, D. R., Dux, P. E., Travis, S. L., \& Mattingley, J. B. (2014). Neural responses to target features outside a search array are enhanced during conjunction but not unique-feature search. Journal of Neuroscience, 34, 3390-3401.

R Core Team. (2012). R: A language and environment for statistical computing. Vienna: R Foundation for Statistical Computing.

Reynolds, J. H., \& Heeger, D. J. (2009). The normalization model of attention. Neuron, 61, 168-185.
Störmer, V. S., \& Alvarez, G. A. (2014). Feature-based attention elicits surround suppression in feature space. Current Biology, 24, 1985-1988.

Treisman, A. M., \& Gelade, G. (1980). A feature-integration theory of attention. Cognitive Psychology, 12, 97-136.

Treue, S., \& Martinez-Trujillo, C. M. (1999). Feature-based attention influences motion processing gain in macaque visual cortex. Nature, 399, 575-579.

Wagner, G., \& Boynton, R. M. (1972). Comparison of four methods of heterochromatic photometry. Journal of the Optical Society of America, 62, 1508-1515.

White, A. L., \& Carrasco, M. (2011). Feature-based attention involuntarily and simultaneously improves visual performance across locations. Journal of Vision, 11, 15.

Wickens, T. D. (2002). Elementary signal detection theory. New York: Oxford University Press.

Wolfe, J. M. (1994). Guided search 2.0: A revised model of visual search. Psychonomic Bulletin \& Review, 1, 202-238.

Zhang, W., \& Luck, S. J. (2009). Feature-based attention modulates feedforward visual processing. Nature Neuroscience, 12, 24-25. 